\title{
AGM Contraction and Revision of Rules
}

\author{
Guido Boella ${ }^{1}$ - Gabriella Pigozzi ${ }^{2}$. \\ Leendert van der Torre ${ }^{3}$
}

\begin{abstract}
In this paper we study AGM contraction and revision of rules using input/output logical theories. We replace propositional formulas in the AGM framework of theory change by pairs of propositional formulas, representing the rule based character of theories, and we replace the classical consequence operator $C n$ by an input/output logic. The results in this paper suggest that, in general, results from belief base dynamics can be transferred to rule base dynamics, but that a similar transfer of AGM theory change to rule change is much more problematic. First, we generalise belief base contraction to rule base contraction, and show that two representation results of Hansson still hold for rule base contraction. Second, we show that the six socalled basic postulates of AGM contraction are consistent only for some input/output logics, but not for others. In particular, we show that the notorious recovery postulate can be satisfied only by basic output, but not by simple-minded output. Third, we show how AGM rule revision can be defined in terms of AGM rule contraction using the Levi identity. We highlight various topics for further research.
\end{abstract}

Gabriella Pigozzi

gabriella.pigozzi@dauphine.fr

Guido Boella

boella@di.unito.it

Leendert van der Torre

leon.vandertorre@uni.lu

1 Università degli Studi di Torino, Torino, Italy

2 CNRS, LAMSADE, Université Paris-Dauphine, PSL Research University, 75016 Paris, France

3 Computer Science and Communication, University of Luxembourg, Esch-sur-Alzette, Luxembourg 
Keywords AGM theory change - Rule based systems - Knowledge representation . Normative systems $\cdot$ Belief revision

\section{Introduction}

Consider a rule based system from which we derive too much, such that we want to weaken it. The following example illustrates how we can use the theory of belief base dynamics (Hansson 1993), based on a suitable notion of consistency, to select a subset of the rules as the contraction of the rule based system.

Example 1 Consider a rule based system consisting of the following two rules:

1. If $a$ then $b$

2. If $b$ then $c$

Assume we do not want to have $c$ in context $\{a\}$, whereas $c$ can be derived by iteratively applying the first and the second rule. We can define rule base contraction operators that drop either the first or the second rule, or both.

We show in this paper how to adapt the theory of belief dynamics (Hansson 1993) to define contraction operators that select some of the rules. This enables us to incorporate various results of belief base dynamics in the area of rule dynamics. However, the following example illustrates that such rule contraction operators may not be sufficient.

Example 2 (Continued) Assume $d$ is an exception to $c$ in context $a$. In that case, we may want to end up with a rule base consisting of the following two rules:

1. If $a \wedge \neg d$ then $b$, and

2. If $b$ then $c$

or a rule base consisting of the following two rules:

1. If $a$ then $b$, and

2. If $b \wedge \neg d$ then $c$.

In other words, in some applications, we may need to change some of the rules. In particular, rule contraction may assume a rule logic which informs us that the rule if $a$ then $b$ ' implies the rule 'if $a \wedge \neg d$ then $b$ ', or that 'if $b$ then $c$ ' implies the rule 'if $b \wedge \neg d$ then $c$.'

In this paper we take AGM theory change as a framework to evaluate the dynamics of rule based systems. AGM theory change is the formal study of how a set of propositions changes in view of a new information that may cause an inconsistency with the existing theory. Expansion, revision and contraction are the three theory change operations that Alchourrón, Gärdenfors and Makinson identified in their approach (called AGM). AGM theory change has two roots, one in counterfactuals by Gärdenfors (1978), and one in the change of legal code by Alchourrón and Makinson (1981, 1982). Although Alchourrón and Makinson aim at defining change operators for a set of norms of some legal system, the condition they impose on a theory is that it is a non-empty and finite set of propositions. In other words, a norm $x$ is taken to be simply a formula in 
propositional logic. Thus, they suggest that "the same concepts and techniques may be taken up in other areas, wherever problems akin to inconsistency and derogation arise" (Alchourrón and Makinson 1981, p. 147). This explains how the work (together with Gärdenfors' analysis of counterfactuals) could ground that research area that is now known as belief revision.

Research question. How to generalise AGM theory change to rule change?

Our research question breaks down into the following three questions:

1. How to apply the Hansson's belief base postulates for contraction and revision to a set of rules?

2. How to apply the AGM belief change postulates for contraction to a set of rules?

3. How to apply the AGM belief change postulates for revision to a set of rules?

As illustrated by Example 2, in general we need a theory in which a set of rules can imply a set of rules, and that can decide whether two sets of rules are equivalent. We do not consider one particular logic for rules, but we consider several of them in the unconstrained input/output logic framework developed by Makinson and Torre (2000). In this theory, we do not necessarily have throughput ("if $p$ then $p$ ") or transitivity, and the framework can therefore be used in a wide range of applications. For example, Makinson and van der Torre observe that in several contexts, one comes across processes resembling inference, but where input propositions are not in general included among outputs, and the operation is not in any way reversible. Examples they give arise in contexts of conditional obligations, goals, ideals, preferences, actions, and beliefs. Their purpose in the unconstrained input/output logic framework is to develop a theory of such input/output operations. We focus on three operations: simple-minded (out 1 ), basic (out 2 , making intelligent use of disjunctive inputs), and simple-minded reusable ( out $_{3}$, in which outputs may be recycled as inputs). Makinson and Torre (2000) define them semantically and characterise them by derivation rules, as well as in terms of relabeling procedures and modal operators. In this paper we consider the contraction and revision of rules in the input/output framework.

Makinson and van der Torre's unconstrained input/output logic has been extended with constraints and applied to normative and legal systems (Makinson and Torre 2001; Parent and Torre 2013). In other words, the unconstrained input/output logics we use in this paper can be used for general input/output processes, of which normative and legal systems are just examples. So the fact that the results hold for general input/output processes, not just for normative or legal systems, could be seen as more general and therefore potentially more interesting. Constrained input/output logic can be used also to define defeasible rules, that is, rules that may have exceptions. Such defeasible rules have been defined, for example, in logic programming using negation as failure. We do not consider defeasible rules or norms in this paper, but we believe that the extension of our results to these other areas is an interesting topic for further research.

The layout of this paper follows the research questions given above and is as follows. In Sect. 2 we introduce our abstract model of normative system change, in Sect. 3 we discuss belief base contraction, in Sect. 4 we discuss AGM contraction and revision, and in Sect. 5 we consider AGM revision for rule based systems. Section 6 refers to related literature and Sect. 7 concludes the paper. 


\section{Abstract Model of the Dynamics of Rule Based Systems}

We first introduce the input/output logic framework, then we discuss how it can be used as a framework for logics for rules, and finally we introduce AGM theory.

\subsection{The Input/Output Logic Framework}

In this paper we start from a general definition of an input/output logic framework introduced by Makinson and Torre (2000), and we discuss several of their principles [for follow-up research in the input/output logic framework the reader is referred to Parent and Torre (2013)].

The first input/output logic principle we adopt in this paper is that rules are represented as pairs of formulas of an arbitrary logic. Here, following most of the work on input/output logic, we use propositional logic for this arbitrary logic, and rules are thus represented by pairs of propositional formulas. The results in this paper hold also if we use a first-order, temporal or action logic, but the use of propositional logic makes the formal exposition simpler, and makes it also straightforward to relate our study to the AGM framework of theory change. The pair of propositional formulas represents a rule, and the two propositional formulas are called the antecedent and consequent of the rule.

Definition 1 (Rules Makinson and Torre 2000) Let $L$ be a propositional logic built on a finite set of propositional atoms $A$. A rule based system $R \subseteq L \times L$ is a set of pairs of $L$, written as $R=\left\{\left(a_{1}, x_{1}\right),\left(a_{2}, x_{2}\right), \ldots,\left(a_{n}, x_{n}\right)\right\}$.

The second principle of the input/output logic framework we adopt in this paper is that the primary role of rules is the derivation of formulas, like the derivation of obligations and prohibitions in a normative system. Which obligations and prohibitions can be derived from a normative system depends on the factual situation, which we call the context or input. We represent the context by a propositional formula. Makinson and van der Torre use a set of propositional formulas as input, but to simplify the third principle in the following section, we assume that the situation can be represented by a finite set of atomic propositions, and thus by their conjunction. The function that associates with each context the set of formulas describes the meaning of the rule based system, because it is a kind of 'operational semantics' of the rule based system. We put the term 'operational semantics' in quotes, because this terminology is not used by Makinson and van der Torre. We use this terminology because we hope it clarifies the notion of 'implied rule' in Definition 6 below. We represent the set of derived formulas as a set of propositional formulas, because that is the simplest representation and it facilitates the comparison with the AGM framework.

Definition 2 ('Operational semantics' Makinson and Torre 2000) An input/output operation out : $\mathcal{P}(L \times L) \times L \rightarrow \mathcal{P}(L)$ is a function from the set of rule based systems and contexts to a set of sentences of $L$.

The following property expresses irrelevance of syntactic presentation. 
Definition 3 (Irrelevance of syntax) out satisfies irrelevance of syntax if the following two properties hold:

IS1 If $x \in \operatorname{out}(R, a), a$ is logically equivalent to $b$ in propositional logic, and $x$ is logically equivalent to $y$, then $y \in \operatorname{out}(R, b)$.

IS2 If $x \in \operatorname{out}(R \cup\{(b, y)\}, a), b$ is logically equivalent to $c$ in propositional logic, and $y$ is logically equivalent to $z$, then $x \in \operatorname{out}(R \cup\{(c, z)\}, a)$.

The simplest input/output logic defined by Makinson and van der Torre is so-called simple-minded output.

Definition 4 (Simple-minded output Makinson and Torre 2000) $x$ is in the simpleminded output of $R$ in context $a$, written as $x \in \operatorname{out}_{1}(R, a)$, if there is a set of rules $\left(a_{1}, x_{1}\right), \ldots,\left(a_{n}, x_{n}\right) \in R$ such that $a_{i} \in C n(a)$ and $x \in C n\left(x_{1} \wedge \ldots \wedge x_{n}\right)$, where $C n(a)$ is the consequence set of $a$ in $L$.

In this paper we use the following running example on the welfare policies of a virtual community. This is an example from normative or legal systems, where the rules can be read as conditional obligations. However, we do not want to suggest that our results hold for such rules only. For example, one can construct as well examples using classification rules, default rules or rules in expert systems. Moreover, as already mentioned in the Introduction, when using input/output logic for normative reasoning, we need to introduce constrained output to deal with contrary-to-duty reasoning (Makinson and Torre 2001). As these concerns are orthogonal to the research questions of this paper, we do not consider constrained output here.

Example 3 (Virtual community) Let the rule based system consist of two rules $R=$ $\{($ poor, house $),($ old, healthins $)\}$, stating that the community has to give a house with low rent (house) to low income agents (poor), and to provide free health insurance (healthins) to elderly agents (old). If no-income implies poor, then the community has to provide a house to someone with no income, represented by the expression house $\in$ out $_{1}(R,($ no - income $\rightarrow$ poor $) \wedge$ no - income $)$, because we have the expressions poor $\in C n(($ no - income $\rightarrow$ poor $) \wedge$ no - income $))$ and house $\in C n$ (house $)$. Moreover, the obligations of the community for low income elderly agents are all logical consequences of giving a house with low rent and providing a free health insurance, because we have $\operatorname{out}_{1}(R$, poor $\wedge$ old $)=C n$ (house $\wedge$ healthins $)$.

The operational semantics of a set of rules can be used to define the two fundamental concepts of equivalence of rule based systems, and the redundancy of a rule in a rule based system. For example, since rule based systems tend to grow quickly and become difficult to understand, redundancy is a useful notion to simplify rule based systems. For systems such as simple-minded output in Definition 4, the following definition is uncontroversial.

Definition 5 (Equivalence and redundancy) Rule based systems $R$ and $S$ are equivalent if and only if for all propositional formulas $a$, we have that $\operatorname{out}(R, a)=\operatorname{out}(S, a)$. A rule $(a, x) \in R$ is redundant in rule based system $R$ if and only if $R$ is equivalent to $R \backslash\{(a, x)\}$.

For more complex rule based systems, more sophisticated notions of equivalence can be considered as well. 


\section{2 'Implication’ Among Rules}

The third principle we adopt from the input/output logic framework is that we define a notion of 'implication' among rules - again we write 'implication' in quotes, because Makinson and van der Torre do not use this terminology. It is not defined as a primitive notion, but derived from the 'operational semantics' of the set of rules. In a rule based system, a set of rules 'implies' another rule $(a, x)$ if and only if $x$ is in the output in context $a$. Likewise, we say that $\langle a, x\rangle$ is implied if $x$ is not in the output in context $a$. For example, the latter corresponds to a weak permission of $\neg x$, which is derived from regulative norms only, not from permissive norms (see Makinson and Torre 2003 for a discussion on this distinction between weak and strong permission in the context of input/output logic).

Definition 6 (Rule 'implication' Makinson and Torre 2000, 2003) Rule $(a, x)$ is 'implied' by rule based system $R$, written as $(a, x) \in \operatorname{out}(R)$, if and only if $x \in \operatorname{out}(R, a)$, and $\langle a, x\rangle$ is 'implied' by $R$, written as $\langle a, x\rangle \in \operatorname{out}(R)$ if and only if $x \notin \operatorname{out}(R, a)$. If it is clear from content which input/output logic out is used, then we write also $\bar{R}$ for $\operatorname{out}(R)$.

Makinson and van der Torre use this new representation of rule 'implication', because it shortens the notation in the definitions of the proof theory, as illustrated in the following example. In this paper we use this notation to enable the use of AGM revision postulates to input/output logics, because we will replace the use of the consequence operator of propositional logic by the out $(R)$ operator. For example, with a notion of implication defined among rules, we can define a success postulate on rule contraction by saying that the new set of rules does not 'imply' the contracted rule (see Sect. 4.4 for the formal details).

Example 4 Strengthening of the Input is represented by out $(R, a) \subseteq$ out $(R, a \wedge b)$, which is equivalent to $x \in \operatorname{out}(R, a)$ implies $x \in \operatorname{out}(R, a \wedge b)$, and which therefore can be represented by: if $(a, x) \in \operatorname{out}(R)$, then $(a \wedge b, x) \in \operatorname{out}(R)$. Visualized as proof rules, this property can thus be represented either by the following rule on the left using the 'operational semantics', or the rule with the rule 'implication' on the right. If the set of rules $R$ does not change, then the two notations are equivalent. We abbreviate Strengthening of the Input by SI.

$$
\frac{x \in \operatorname{out}(R, a)}{x \in \operatorname{out}(R, a \wedge b)} S I \quad \frac{(a, x)}{(a \wedge b, x)} S I
$$

Yet another way to represent this principle is the contrapositive: if $(a \wedge b, x) \notin \operatorname{out}(R)$, then $(a, x) \notin \operatorname{out}(R)$, which can be represented by the following two equivalent representations of the Weakening of the Input or WI proof rule. It may be seen as the inverse of the Strengthening of the Input rule, and Makinson and van der Torre therefore write also $S I^{-1}$.

$$
\frac{x \notin \operatorname{out}(R, a \wedge b)}{x \notin \operatorname{out}(R, a)} S I^{-1}=W I \quad \frac{\langle a \wedge b, x\rangle}{\langle a, x\rangle} S I^{-1}=W I
$$


Other properties can be represented as proof rules too. For example, the first item of irrelevance of syntax or IS1 in Definition 3 can be represented as follows, where $\models$ stands for logical implication in propositional logic.

$$
\frac{x \in \operatorname{out}(R, a), \models a \leftrightarrow b, \models x \leftrightarrow y}{y \in \operatorname{out}(R, b)} I S 1 \quad \frac{(a, x), \models a \leftrightarrow b, \models x \leftrightarrow y}{(b, y)} I S 1
$$

Thus, to decide when a set of rules 'implies' another rule, we resort to the 'operational semantics' of the rules.

\subsection{Closure Properties on Rules}

Makinson and van der Torre observe that the relation between the 'implication' among rules $(a, x) \in \operatorname{out}(R)$ and the 'operational semantics' $x \in \operatorname{out}(R, a)$ has an analogy in classical logic, where the pair $a \models x$ is equivalent to the membership of $x$ in the consequence set of $a$, written as $x \in C n(a)$. However, it is important to see that the notion of 'implication' should not be identified with the notion of implication among conditionals in classical conditional logic see, e.g., Nute (1984). This is illustrated by the Tarskian properties of a consequence operation, i.e. the closure operation on the input/output pairs. Whereas these properties normally hold for a classical conditional logic, they represent non-trivial principles for a logic of rules. These additional principles may either be accepted or rejected.

Reflexivity expresses that if the input is precisely the antecedent of one of the rules, then the output contains the consequent of the rule. Monotony expresses that the application of one rule cannot block another rule, as in most rule based non-monotonic logics like, for example, Reiter's default logic. Idempotence expresses that if we have $x$ in the output of $a$, then we can add $(a, x)$ as a rule without changing the output.

Definition 7 (Closure) out is a closure operation when the following three conditions hold:

Reflexivity. $x \in$ out $(R \cup\{(a, x)\}, a)$ (in other words, $R \subseteq$ out $(R))$, if the context is precisely the antecedent of one of the rules, then the output contains the consequent of that rule.

Monotony. $x \in \operatorname{out}\left(R_{1}, a\right)$ implies $x \in \operatorname{out}\left(R_{1} \cup R_{2}, a\right)$ (in other words, $\left.\operatorname{out}\left(R_{1}\right) \subseteq \operatorname{out}\left(R_{1} \cup R_{2}\right)\right)$, if the set of rules increases, then no conclusions are lost.

Idempotence. if $x \in \operatorname{out}(R, a)$, then for all $b$, we have $\operatorname{out}(R, b)=\operatorname{out}(R \cup$ $\{(a, x)\}, b)$ (in other words, out $(R)=\operatorname{out}($ out $((R)))$, if $x$ is obligatory in context $a$, then $(a, x)$ can be added to the rule based system without changing the output.

Makinson and van der Torre show that their seven input/output logics satisfy the Tarskian properties, and their notion of 'implication' among rules is therefore a Tarskian consequence relation. In this paper we consider only the first six of their logics. 
Definition 8 (Makinson and Torre 2000) Let $R(a)=\{x \mid(a, x) \in R\}$, and a complete formula $v$ is a conjunction of a maxiconsistent set of literals, or the conjunction of all literals of $L$. Simple-minded, basic and reusable output are defined as follows.

$$
\begin{aligned}
& \text { out }_{1}(R, a)=C n(R(C n(a))) \\
& \text { out }_{2}(R, a)=\cap\left\{\text { out }_{1}(R, v) \mid a \in C n(v), v \text { complete }\right\} \\
& \text { out }_{3}(R, a)=\cap\left\{\text { out }_{1}(R, b) \mid a \in C n(b), \text { out }_{1}(R, b) \subseteq C n(b)\right\}
\end{aligned}
$$

Moreover, for each input/output logic a corresponding throughput operator is defined by:

$$
\text { out }_{i}^{+}(R, a)=\text { out }_{i}(R \cup\{(b, b) \mid b \in L\}, a)
$$

The following property shows the axiomatisation of the six logics, and implies that none of out 1 , out 2 and out $_{3}$ satisfies identity $((a, a)$ for all $a)$ or contraposition. The throughput operations satisfy identity. Basic output handles reasoning by cases, and reusable output handles iterated detachment. See (Makinson and Torre 2000) for examples and additional properties.

Proposition 1 (Makinson and Torre 2000) out $1(R)$ is the minimal set that contains $R \cup\{(\top, \top)\}$, is closed under replacement of logical equivalents in antecedent and consequent, and the following inference rules strengthening of the input (SI), weakening of the output (WO), and conjunction of output (AND).

$$
\frac{(a, x)}{(a \wedge b, x)} S I \quad \frac{(a, x \wedge y)}{(a, x)} W O \quad \frac{(a, x),(a, y)}{(a, x \wedge y)} A N D
$$

out $_{2}$ is closed in addition under the inference rule disjunction $(O R)$, and out 3 under the inference rule cumulative transitivity $(C T)$. Moreover, out ${ }_{i}^{+}$is closed under the same inference rules as out ${ }_{i}$ together with Identity (Id).

$$
\frac{(a, x),(b, x)}{(a \vee b, x)} \text { OR } \quad \frac{(a, x),(a \wedge x, y)}{(a, y)} C T \quad \frac{-}{(a, a)} I d
$$

To prove Proposition 7 below, it will be useful to use the following observation of Makinson and van der Torre.

Lemma 1 (Makinson and Torre 2003, Observation 3) The non-repetition property says that if there is a derivation of $(a, x)$ from a set of rules $R$, then there is another derivation of $(a, x)$ from $R$, where each rule of $R$ is used at most once. The nonrepetition property holds for:

- out 1 with SI, WO, AND

- out 2 with SI, WO, AND, OR

- out 3 with SI, WO, CTA

- out ${ }_{i}^{+}$with rules of out ${ }_{i}$ together with Id

where CTA derives $(a, x \wedge y)$ from $(a, x)$ and $(a \wedge x, y)$. 


\subsection{AGM Rule Change}

The AGM framework (Alchourrón et al. 1985) for theory revision is the formal study of how a set of propositions should change in view of a new information that may cause an inconsistency with the existing ones. Expansion, revision and contraction are the three theory change operations that Alchourrón, Gärdenfors and Makinson identified in their AGM approach. The initial inspiration was a formal analysis of the dynamics of a legal code. How can a rule be removed or added to a code? What happens when the addition of a new law conflicts with some of the existing ones?

They observed that these operations are more general and can be defined on any deductively closed set $K$ of propositional formulas. In particular, the expansion of $K$ by a formula $x$ is a set that accepts the unproblematic $x$ and it is denoted by $K+x$. A contraction of $K$ by $x$ results in a set $K-x$ from which $x$ does not follow anymore. Finally, the revision of $K$ by $x$ yields to a set $K * x$ which contains $x$ and from which parts of $K$ that were conflicting with $x$ have been removed.

AGM theory is a well established formal theory. Here, we want to take back its original inspiration and use AGM postulates for rule change. Therefore, we define rule expansion, rule revision, and rule contraction. To generalize the AGM postulates for our framework, we define a rule set as a set of rules closed under an input/output logic, denoted by $\bar{R}$. Since we do not want to restrict ourselves to one particular logic, we use out to refer to any input/output logic. We write $\bar{R} \oplus(a, x)$ to indicate the expansion of a rule based system $R$ by a new rule, $\bar{R} \ominus(a, x)$ for the contraction of a rule $(a, x)$ from $\bar{R}$, and $\bar{R} \circledast(a, x)$ for the revision of $\bar{R}$ by the new rule $(a, x)$.

Like AGM expansion, the definition of rule expansion is straightforward. The new rule that the legislator wants to enforce does not cause any conflict with the existing legal code. Hence, $(a, x)$ is added to $\bar{R}$ together with all the rules that can be derived from the union of $\bar{R}$ and $(a, x)$ (similarly to theory revision, we assume $\bar{R}$ to be closed under input/output logic): $\bar{R} \oplus(a, x)=\overline{R \cup\{(a, x)\}}$.

\section{Example $5 \overline{\{(\text { poor }, \text { house })\}} \oplus$ (old, healthins $)=\overline{\{(\text { poor }, \text { house }),(\text { old }, \text { healthins })\}}$}

Theory contraction and revision are the more complex and interesting types of change. The Levi and Harper identities highlight that revision and contraction are interdefinable. More specifically, the Levi identity defines the revision of a set by $x$ as the expansion of the contracted set by $\neg x$. Because of the simplicity of this definition, the contraction is often considered as the basic type of change in revision. For this reason, we also start from rule contraction.

Does revision really offer a satisfactory framework for rule revision? When we consider a set of conditional rules, the AGM principles may prove to be too general to deal with the revision of a rule based system. In order to have a closer look to these questions, we now need to turn to Hansson's postulates for belief base dynamics (Hansson 1993) as well as AGM theory contraction and revision and to the corresponding rule contraction and revision. 


\section{Belief Base Dynamics}

In this section we show that two of Hansson's representation theorems for belief base contraction (Hansson 1993) still hold for rule base contraction, and we discuss topics for further research on rule base dynamics. In this section we repeat several definitions from Hansson's paper, because we want to make it very clear and explicit that the generalisation of two Hansson's representation theorems is straightforward, and where the problems are in generalising some of his other results.

\subsection{Hansson's Representation Theorems for Belief Base Contraction}

In this section we give an overview of some of the definitions and results of Hansson's belief base contraction. We adopt the definitions of Hansson (1993), because in that paper the operators are phrased in terms of a general consequence operator, which can be exchanged with an input/output operator. See the paper of Hansson (1993) for a further discussion and examples.

Definition 9 (Hansson 1993) Let $L$ (the language) be a set of expressions that is closed under truth-functional operations. A consequence operation on $L$ is a function $C n$ from $\mathcal{P}(L)$ to $\mathcal{P}(L)$ such that, for all subsets $A$ and $B$ of $L$ :

1. $A \subseteq C n(A)$

(inclusion)

2. If $A \subseteq B$, then $C n(A) \subseteq C n(B)$

(monotony)

3. $C n(A)=C n(C n(A))$

(iteration)

A subset $A$ of $L$ is consistent if and only if there is no $x \in L$ such that both $x \in \operatorname{Cn}(A)$ and $\neg x \in C n(A)$.

Hansson assumes that the consequence operator includes classical truth-functional logic and satisfies the properties of deduction and compactness. The input/output logics in Definition 8 also satisfy compactness, but in input/output logic there are no analogues for supraclassicality and deduction. We therefore have to check all proofs of Hansson to find out which of his results still hold when we replace propositional sentences by pairs of such sentences.

Postulate 1 (Hansson 1993) Cn satisfies the following three properties:

4. If $x$ can be derived from $A$ by classical truth-functional logic, then $x \in C n(A)$ (supraclassicality)

5. If $y \in C n(A \cup\{x\})$, then $(x \rightarrow y) \in C n(A)$

(deduction)

6. If $x \in C n(A)$, then $x \in C n\left(A^{\prime}\right)$ for some finite subset $A^{\prime}$ of $A$

(compactness).

Hansson adopts the following notation from the AGM literature. $K \perp A$ is the set of inclusion-maximal subsets of $K$ that do not imply any of the elements of $A$.

Definition 10 (Alchourrón and Makinson 1981; Hansson 1993) Let $K$ and $A$ be subsets of $L$. Then $K \perp A$ is the set of all subsets $K^{\prime}$ of $K$ such that $A \cap C n\left(K^{\prime}\right)=\varnothing$ and that there is no set $K^{\prime \prime}$ such that $K^{\prime} \subset K^{\prime \prime} \subseteq K$ and $A \cap C n\left(K^{\prime \prime}\right)=\emptyset$. 
We consider here two of Hansson's representation results. The first takes subsets of the knowledge base, using the inclusion-maximal subsets. In what follows, " " denotes the partial meet contraction determined by a specified selection function $\gamma$, whereas " $\div$ " denotes contraction operators in general. Partial meet contraction selects the intersection of some of the maximal subsets of the belief set not implying the contracted formula.

Definition 11 (Hansson 1993) $\gamma$ is a one-place selection function for the subset $K$ of $L$ iff $\gamma$ is a function from $\mathcal{P} \mathcal{P}(K)$ to $\mathcal{P} \mathcal{P}(K)$, such that for all subsets $A$ of $L, \gamma(K \perp A)$ is a nonempty subset of $K \perp A$, unless $K \perp A$ is empty, in which case $\gamma(K \perp A)=\{K\}$.

Let $\gamma$ be a one-place selection function for the subset $K$ of $L$. Then $\sim_{\gamma}$, the operator of partial meet contraction for $K$ generated by $\gamma$, is the operator such that, for all subsets $A$ of $L$ :

$$
K \sim_{\gamma} A=\cap \gamma(K \perp A) .
$$

Hansson's first representation theorem shows that partial meet contraction operators are characterised by four properties, called inclusion, relevance, success and uniformity.

Theorem 1 (Hansson 1993) Let $\div$ be an operator for a subset $K$ of L. Then $\div$ is an operator of partial meet contraction generated by some one-place selection function for $K$ iff it satisfies the following conditions.

$(\div 1) K \div A \subseteq K$

(inclusion)

$(\div 2)$ If $x \in K \backslash(K \div A)$, then there is some $K^{\prime}$ with $K \div A \subseteq K^{\prime} \subseteq K$, such that: $A \cap C n\left(K^{\prime}\right)=\emptyset$ and $A \cap C n\left(K^{\prime} \cup\{x\}\right) \neq \emptyset$

(relevance)

$(\div 3)$ If $A \cap C n(\emptyset)=\emptyset$, then $A \cap C n(K \div A)=\emptyset$ (success)

$(\div 4)$ If it holds for all subsets $K^{\prime}$ of $K$ that $A \cap C n\left(K^{\prime}\right)=\emptyset$ iff $B \cap C n\left(K^{\prime}\right)=\emptyset$, then: $K \div A=K \div B$

(uniformity)

For the second representation theorem, Hansson defines more general two-place selection functions, which he requires to be unified.

Definition 12 (Hansson 1993) A two-place selection function is a function $\gamma$ such that for each subset $K$ of $L, \gamma(K)=,\gamma_{K}$ () is a one-place selection function for $K$. A two-place selection function $\gamma$ is unified if and only if for all subsets $K_{1}$ and $K_{2}$ of $L$ : if $K_{1} \perp A_{1}=K_{2} \perp A_{2} \neq \emptyset$, then $\cap \gamma_{K_{1}}\left(K_{1} \perp A_{1}\right)=\cap \gamma_{K_{2}}\left(K_{2} \perp A_{2}\right)$.

The first argument of a unified two-place selection function can be omitted. Thus $\cap \gamma(K \perp A)$ is an abbreviation of $\cap \gamma_{K}(K \perp A)$.

An operator $\sim_{\gamma}$ is the operator of partial meet contraction generated by the twoplace selection function $\gamma$ iff, for all subsets $K$ and $A$ of $L: K \sim_{\gamma} A=\cap \gamma_{K}(K \perp A)$.

$\sim_{\gamma}$ is unified iff $\gamma$ is unified.

Hansson's second representation theorem shows that such unified partial meet contraction operators are characterised by one additional property, called redundancy.

Theorem 2 (Hansson 1993) An operator $\div$ is an operator of unified partial meet contraction iff it satisfies conditions $(\div 1)-(\div 4)$ and in addition the following:

$(\div 5)$ If $A \cap C n(\emptyset)=\varnothing$, and each element of $Z$ implies an element of $A$, then: $K \div A=(K \cup Z) \div A$

(redundancy) 


\subsection{Rule Base Contraction}

The definitions of rule base contraction are straightforward: we simply replace the knowledge base by a rule base, and we replace $C n$ by out. $R \perp A$ is the set of inclusionmaximal subsets of $R$ that do not imply any of the elements of $A$. " " denotes the partial meet contraction determined by a specified selection function $\gamma$, whereas " $\div$ " denotes contraction operators in general.

Definition 13 Let $A, R$ be sets of pairs of $L$. Then $R \perp A$ is the set of all subsets $R^{\prime}$ of $R$ such that $A \cap \operatorname{out}\left(R^{\prime}\right)=\emptyset$ and that there is no set $R^{\prime \prime}$ such that $R^{\prime} \subset R^{\prime \prime} \subseteq R$ and $R \cap \operatorname{out}\left(R^{\prime \prime}\right)=\emptyset$.

Definition $14 \gamma$ is a one-place selection function for the set $R$ of pairs of $L$ iff $\gamma$ is a function from $\mathcal{P} \mathcal{P}(R)$ to $\mathcal{P} \mathcal{P}(R)$, such that for all subsets $A$ of $L, \gamma(R \perp A)$ is a nonempty subset of $R \perp A$, unless $R \perp A$ is empty, in which case $\gamma(R \perp A)=\{R\}$.

Let $\gamma$ be a one-place selection function for the set $R$ of pairs of $L$. Then $\sim_{\gamma}$, the operator of partial meet contraction for $R$ generated by $\gamma$, is the operator such that, for all sets $A$ of pairs of $L$ :

$R \sim_{\gamma} A=\cap \gamma(R \perp A)$.

Theorem 3 Let $\div$ be an operator for a subset $R$ of $L$. Then $\div$ is an operator of partial meet contraction generated by some one-place selection function for $R$ iff it satisfies the following conditions.

$(\div 1) R \div A \subseteq R$

(inclusion)

$(\div 2)$ If $x \in R \backslash(R \div A)$, then there is some $R^{\prime}$ with $R \div A \subseteq R^{\prime} \subseteq R$, such that: $A \cap C n\left(R^{\prime}\right)=\emptyset$ and $A \cap C n\left(R^{\prime} \cup\{x\}\right) \neq \emptyset$

(relevance)

$(\div 3)$ If $A \cap C n(\emptyset)=\emptyset$, then $A \cap$ out $(R \div A)=\emptyset$

(success)

$(\div 4)$ If it holds for all subsets $R^{\prime}$ of $R$ that $A \cap$ out $\left(R^{\prime}\right)=\emptyset$ iff $B \cap$ out $\left(R^{\prime}\right)=\emptyset$, then: $R \div A=R \div B$

(uniformity)

Proof The proof is analogous to Hansson (1993)'s proof of Theorem 1, replacing $K$ by $R$ and replacing $C n$ by out.

Likewise, the definitions for unified contraction can directly be taken from Hansson.

Definition 15 A two-place selection function is a function $\gamma$ such that for each subset $R$ of $L, \gamma(R)=,\gamma_{R}()$ is a one-place selection function for $R$. A two-place selection function $\gamma$ is unified if and only if for all subsets $R_{1}$ and $R_{2}$ of $L$ : if $R_{1} \perp A_{1}=$ $R_{2} \perp A_{2} \neq \emptyset$, then $\cap \gamma_{R_{1}}\left(R_{1} \perp A_{1}\right)=\cap \gamma_{R_{2}}\left(R_{2} \perp A_{2}\right)$.

The first argument of a unified two-place selection function can be omitted. Thus $\cap \gamma(R \perp A)$ is an abbreviation of $\cap \gamma_{R}(R \perp A)$.

An operator $\sim_{\gamma}$ is the operator of partial meet contraction generated by the twoplace selection function $\gamma$ iff, for all sets $R$ and $A$ of pairs of $L: \sim_{\gamma} A=\cap \gamma_{R}(R \perp A)$. $\sim_{\gamma}$ is unified iff $\gamma$ is unified.

Moreover, the proof of the second theorem goes through as well.

Theorem 4 An operator $\div$ is an operator of unified partial meet contraction iff it satisfies conditions $(\div 1)-(\div 4)$ and in addition the following: 
$(\div 5)$ If $A \cap$ out $(\emptyset)=\emptyset$, and each element of $Z$ implies an element of $A$, then: $R \div A=(R \cup Z) \div A$

(redundancy)

Proof The proof is analogous to Hansson (1993)'s proof of Theorem 2, replacing $K$ by $R$ and replacing $C n$ by out.

\subsection{Further Research: Rule Base Revision}

The positive results do not imply that all of Hansson's results can directly be transferred to rule base dynamics. For example, the following third result of Hansson refers to a conjunction of formulas, which is not defined for input/output logics (see his paper for the definitions of maximizingly transitively relational partial meet contraction). This issue is analogous to the problem of representing the seventh and eight postulate of AGM contraction in rule contraction, which we discuss in the following section.

Theorem 5 (Hansson 1993) The following condition

$$
(K \div\{p\}) \cap(K \div\{q\}) \subseteq K \div\{p \& q\}
$$

(intersection)

(1) holds for all operators of maximizingly, transitively relational partial meet contraction, but (2) does not hold in general for operators of transitively relational partial meet contraction.

Moreover, Hansson distinguishes internal from external revision, which he defines using the following function.

Definition 16 (Hansson 1993) Let $A$ be a finite subset of $L$. Then $n(A)$ is defined as follows:

1. $n(\emptyset)=\perp$, where $\perp$ is a contradiction.

2. If $A=\left\{p_{1}, p_{2}, \ldots, p_{m}\right\}$ for some $m \leq 1$, then: $n(A)=\neg p_{1} \vee \ldots \vee \neg p_{m}$

Let $\gamma$ be a one-place selection function for the subset $K$ of $L$. Then the operator $*_{\gamma}$ is the operator of internal partial meet revision for $K$ generated by $\gamma$ iff for all finite subsets $A$ of $L$ :

$$
K *_{\gamma} A=\cap \gamma(K \perp\{n(A)\}) \cup A .
$$

Let $\gamma$ be a two-place selection function. The operator of external partial meet revision generated by $\gamma$ is the operator \pm such that for all subsets $K$ of $L$ and all finite subsets $A$ of $L$ :

$$
K \pm A=\cap \gamma_{K \cup A}((K \cup A) \perp\{n(A)\}) .
$$

Thus, to define the corresponding rule revision operators, we need to define the negation $n(R)$ of a set of rules $R$ (see, e.g., Governatori and Rotolo 2010; Governatori et al. 2013). In some other papers on belief base dynamics, see for example the survey by Gärdenfors and Rott (1995), belief base revision is defined for contraction and revision by a single formula only. For the corresponding rule revision we need to define at least the negation of a rule. We do not further pursue these questions here, but turn now to AGM contraction operators. 


\section{AGM Rule Contraction}

We start by reminding the AGM contraction postulates.

Definition 17 (Alchourrón et al. 1985) Let $K$ be a deductively closed set of propositional formulas. An AGM contraction operation - satisfies the following postulates.

$\mathrm{K}-1: K-x$ is a deductively closed set

$\mathrm{K}-2: K-x \subseteq K$

K-3: If $x \notin K$ then $K=K-x$

(closure or type)

K-4: If $\nvdash x$ then $x \notin(K-x)$

(inclusion or contraction)

K-5: If $x \in K$ then $K \subseteq(K-x)+x$

(vacuity or min. action)

K-6: If $\vdash x \leftrightarrow y$ then $\bar{K}-x=K-y$

(success)

(recovery)

K-7: $((K-x) \cap(K-y)) \subseteq K-(x \wedge y)$

(extensionality)

K-8: If $x \notin(K-(x \wedge y))$ then $K-(x \wedge y) \subseteq K-x$

(min-conjunction)

(max-conjunction)

The first problem we encounter is that the last two postulates refer to conjunctions, which are not defined for rules. This is not a major problem, since the first six postulates of AGM theory are known as the basic postulates, whereas the latter two are optional. So we restrict ourselves to the basic postulates. ${ }^{1}$

Definition 18 Let out be an input/output logic. A rule contraction operator $\ominus$ satisfies the following postulates.

$\mathrm{R}-1: \bar{R} \ominus(a, x)$ is closed under out

$\mathrm{R}-2: \bar{R} \ominus(a, x) \subseteq \bar{R}$

R-3: If $(a, x) \notin \bar{R}$ then $\bar{R}=\bar{R} \ominus(a, x)$

(closure or type)

$\mathrm{R}-4:$ If $(a, x) \notin$ out $(\emptyset)$ then $(a, x) \notin \bar{R} \ominus(a, x)$

(vacuity or min. action)

R-5: If $(a, x) \in \bar{R}$ then $\bar{R} \subseteq(\bar{R} \ominus(a, x)) \oplus(a, x)$

(success)

(recovery)

R-6: If $\operatorname{out}(\{(a, x)\})=\operatorname{out}(\{(b, y)\})$ then $\bar{R} \ominus(a, x)=\bar{R} \ominus(b, y)$ (extensionality)

In the remainder of this section, we are going to study these postulates. We approach them by translating the 'implied rule' representation back to the 'operational semantics'.

\subsection{R-1: Closure}

$\mathrm{K}-1: K-x$ is a deductively closed set

(closure or type)

$\mathrm{R}-1: \bar{R} \ominus(a, x)$ is closed under out

(closure or type)

The first postulate for theory change requires that the result of the contraction is a deductively closed set. Expansion, contraction and revision operators are indeed defined as functions from a deductively closed set and a formula $x$ to a new deductively closed set.

The corresponding closure (type) postulate for rule contraction says that $\bar{R} \ominus(a, x)$ is closed under out. In other words, if $(b, y) \in \operatorname{out}(\bar{R} \ominus(a, x))$, then $(b, y) \in \bar{R} \ominus(a, x)$. Therefore, if $y \in \operatorname{out}(\bar{R} \ominus(a, x), b)$, then $(b, y) \in \bar{R} \ominus(a, x)$.

\footnotetext{
${ }^{1}$ K-7 and K-8 can be formulated on contraction with a set of formulas, so, the contraction of $a \wedge b$ can be understood as the contraction of $\{a, b\}$ see, e.g., Billington and Antoniou (1999).
} 


\subsection{R-2: Inclusion}

$\mathrm{K}-2: K-x \subseteq K$

$\mathrm{R}-2: \bar{R} \ominus(a, x) \subseteq \bar{R}$

(inclusion or contraction)

(inclusion or contraction)

K-2 states that, because $K-x$ is obtained from $K$ by giving up $x$, it is required that no new formulas occur in $K-x$.

The corresponding inclusion (contraction) postulate for rule contraction says that $\bar{R} \ominus(a, x) \subseteq \bar{R}$. Together with R-1, this property says that if $y \in \operatorname{out}(\bar{R} \ominus(a, x), b)$, then $y \in \operatorname{out}(\bar{R}, b)$. In other words, for any context $b$, if something is obligatory after rule contraction, then it was already obligatory before the contraction.

\subsection{R-3: Vacuity}

K-3: If $x \notin K$ then $K=K-x$

R-3: If $(a, x) \notin \bar{R}$ then $\bar{R}=\bar{R} \ominus(a, x)$

(vacuity or min. action) (vacuity or min. action)

If $x \notin K$, the criterion of minimal change requires that nothing is retracted from $K$. The corresponding vacuity (min. action) postulate for rule contraction says that if $(a, x) \notin \bar{R}$ then $\bar{R}=\bar{R} \ominus(a, x)$. Together with $\mathrm{R}-1$, this property says that if $x \notin \operatorname{out}(\bar{R}, a)$, then $\bar{R}=\bar{R} \ominus(a, x)$. In other words, if we contract a rule $(a, x)$, but $x$ is not obligatory in context $a$, then the contraction does not have any effect.

\subsection{R-4: Success}

K-4: If $\nvdash x$ then $x \notin(K-x)$

R-4: If $(a, x) \notin \operatorname{out}(\emptyset)$ then $(a, x) \notin \bar{R} \ominus(a, x)$

K-4 says that, unless $x$ is logically valid (in which case it can never be retracted), if we remove $x$ from $K$, the resulting set will not contain $x$.

The corresponding success postulate for rule contraction says that if $(a, x) \notin \operatorname{out}(\emptyset)$ then $(a, x) \notin \bar{R} \ominus(a, x)$. In other words, if $x \notin \operatorname{out}(\emptyset, a)$, then $x \notin \operatorname{out}(R \ominus(a, x), a)$. The following example illustrates the success postulate.

Example 6 Assume out $1, R=\{($ poor, house $\wedge$ healthins $)\}$ and four possible rule based systems $\bar{R} \ominus$ (poor $\wedge$ old, house) visualized in Fig. 1. This figure must be read as follows. Each column represents one possible way in which (poor $\wedge$ old, house) can be contracted from $\bar{R}$. The short notation is on top, and the output of the set of rules for each input on the left is written below it.

The success condition says that house $\notin$ out $\left(\bar{R}^{\prime}\right.$, poor $\wedge$ old $)$, which is not satisfied by $\overline{R_{1}}$, but it is satisfied by $\overline{R_{2}}, \overline{R_{3}}$ and $\overline{R_{4}}$. Thus, there are several ways in which a set of rules can be contracted. The purpose of the postulates is precisely to distinguish the admissible solutions from the inadmissible ones.

However, unlike in AGM theory revision, the question here is not only what and how much to contract, but also for which inputs to contract. This is what distinguishes the different solutions in the example. All the possible $\overline{R_{i}}$ prescribe that a free health 


\begin{tabular}{|c|c|c|c|c|}
\hline & $\frac{\bar{R}=\overline{R_{1}}=}{\{(p r, h s e \wedge h i)\}}$ & $\frac{\overline{R_{2}}=}{\{(p r, h i)\}}$ & $\frac{\overline{R_{3}}=}{\frac{\{(p r, h i),}{(p r \wedge \neg o l d, h s e \wedge h i)\}}}$ & 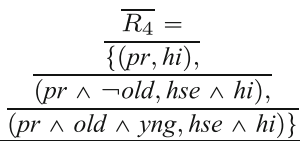 \\
\hline$T$ & $C n(\top)$ & $C n(\top)$ & $C n(\top)$ & $C n(\top)$ \\
\hline$p r$ & Cn $(h s e \wedge h i)$ & $C n(h i)$ & $C n(h i)$ & $C n(h i)$ \\
\hline$\neg p r$ & $C n(\top)$ & $C n(\top)$ & $C n(\top)$ & $C n(\top)$ \\
\hline$p r \vee$ old & $C n(\top)$ & $C n(\top)$ & $C n(\top)$ & $C n(\top)$ \\
\hline$p r \wedge$ old & $C n(h s e \wedge h i)$ & $C n(h i)$ & $C n(h i)$ & $C n(h i)$ \\
\hline$p r \wedge \neg$ old & Cn $(h s e \wedge h i)$ & $C n(h i)$ & $C n(h s e \wedge h i)$ & $C n(h s e \wedge h i)$ \\
\hline$p r \wedge$ old $\wedge y n g$ & $C n(h s e \wedge h i)$ & $C n(h i)$ & $C n(h i)$ & $C n(h s e \wedge h i)$ \\
\hline
\end{tabular}

Fig. 1 Four alternatives for $\bar{R} \ominus(p r \wedge$ old, hse $), \overline{R_{2}}, \overline{R_{3}}$ and $\overline{R_{4}}$ satisfy the success postulate. In the figure we use the following abbreviations: low rent (hse), low income agents $(p r)$, free health insurance $(h i)$, elderly agents (old), and young agents (yng)

insurance must be given to the poor. Independently of whether the input is the poor people only, or the poor and old or, again, the poor and the not old, we obtain the same $\overline{R_{i}}$. Hence, in order to obtain a rule based system that performs a contraction for all poor people (also the not old ones), $\overline{R_{i}}$ should contain an additional rule as in $R_{3}$ and $R_{4}$.

We now consider the question of characterising the minimal rule contraction operators, in other words, how to keep as much of possible of $\bar{R}$ in $\bar{R} \ominus(a, x)$. Though this construction is considered to have implausible properties, it illustrates some of the challenges in rule contraction. In AGM, the minimal contraction of $x$ from $K$ selects one of the maximal subsets of $K$ consistent with $x$, which can be characterised as $C n(\{x \rightarrow y \mid y \in K\})$, where $y$ is a consistent complete formula (i.e., a conjunction of a maxiconsistent set of literals) not implying $x$. However, no such simple syntactic characterisation seems to be available for minimal rule contraction.

The source of the problem can be illustrated as follows. In AGM, if $y \notin(K-x)$, then we also have $x \wedge y \notin(K-x)$. This follows directly from the closure postulate $\mathrm{K}-1$, i.e. from the fact that belief sets are closed under consequence. Likewise, if $(a, x) \notin \bar{R} \ominus(a, x)$, then also $(a, x \wedge y) \notin \bar{R} \ominus(a, x)$. However, this is not the only consequence of the success postulate for rule contraction. For example, for all six input/output logics we consider in this paper, if $(a, x) \notin \bar{R} \ominus(a, x)$, then also $(a \vee b, x) \notin \bar{R} \ominus(a, x)$.

Other logical relations depend on the input/output logic used. For example, for basic output out $_{2}$, if $(a, x) \notin \bar{R} \ominus(a, x)$, then we have either $(a \wedge b, x) \notin \bar{R} \ominus(a, x)$ or $(a \wedge \neg b, x) \notin \bar{R} \ominus(a, x)$. In other words, if $(a, x) \notin \bar{R} \ominus(a, x)$ and $(a \wedge b, x) \in$ $\bar{R} \ominus(a, x)$, then $(a \wedge \neg b, x) \notin \bar{R} \ominus(a, x)$. These relations do not hold for simpleminded output out $_{1}$. Likewise, a similar property based on the inverse of CTA holds for reusable output $\mathrm{out}_{3}$.

Example 7 Consider a finite set of propositional atoms. In AGM, a minimal contraction operator $K-x$ is the set of formulas $C n(\{z \vee y) \mid z \in K\})$, where $y$ is a consistent complete formula not implying $x$. Likewise, for out $_{1}$, the minimal contraction operators gives for $\bar{R} \ominus(a, x)$ the set of rules $\operatorname{out}(\{(b, z \vee y) \mid a \models b,(b, z) \in$ 
$\bar{R}\}) \cup \operatorname{out}(\{(b, z) \mid b \not \models a,(b, z) \in \bar{R}\})$, where $y$ is a consistent complete formula not implying $x$. This follows from non-repetition property in Lemma 1.

In Definition 6 we mentioned that we also write $\langle a, x\rangle \in \bar{R}$ for $(a, x) \notin \bar{R}$. Thus, we can also write the success postulate as:

R-4: If $\langle a, x\rangle \in \operatorname{out}(\emptyset)$ then $\langle a, x\rangle \in \bar{R} \ominus(a, x)$

(success)

\subsection{R-5: Recovery}

K-5: If $x \in K$ then $K \subseteq(K-x)+x$

(recovery)

R-5: If $(a, x) \in \bar{R}$ then $\bar{R} \subseteq(\bar{R} \ominus(a, x)) \oplus(a, x)$

(recovery)

K-5 requires that expanding $K-x$ by $x$ should give the same set as before the contraction, that is $K$.

The corresponding recovery postulate for rule contraction says that, if $(a, x) \in \bar{R}$, then $\bar{R} \subseteq(\bar{R} \ominus(a, x)) \oplus(a, x)$. In other words, contracting a rule based system by $(a, x)$ and then expanding by the same $(a, x)$ should leave $\bar{R}$ unchanged.

Example 8 Assume $R=\{($ poor, house $\wedge$ healthins $),($ old, house $\wedge$ healthins $)\}$ and $\bar{R}^{\prime}=\bar{R} \ominus$ (poor, house). Suppose that $\bar{R}^{\prime}$ is calculated by the obviously too strong principle that house is contracted from the consequent of each rule, such that $\bar{R}^{\prime}=$ out $(\{($ poor, healthins $),($ old, healthins $)\})$. This does not satisfy recovery since for all reasonable input/output logics, adding (poor, house) to $\bar{R}^{\prime}$ does not give us $\bar{R}$ again.

The following result shows that the five postulates considered thus far are consistent only for some input/output logics, but not for others. In particular, if we adopt out $_{1}$ or out $_{3}$ then there is no single rule contraction operator satisfying the postulates.

Proposition 2 R-1 until R-5 cannot hold together for out ${ }_{1}$ or out $_{3}$, but they can hold together for out 2 .

Proof A counterexample for out 1 and out 3 is given by rule based system $R=$ $\{($ poor, house $)\}$. Now contract (poor, house) from $\bar{R}$. The recovery postulate requires (poor, house $) \notin(\{($ poor, house $)\} \ominus($ poor $\wedge$ old, house $)) \oplus($ poor $\wedge$ old, house $)$. (poor, house $)$ is not part of $\{($ poor, house $)\} \ominus$ ( poor $\wedge$ old, house), and there is no way to derive it when ( poor $\wedge$ old, house) is added again. The same counterexample also works for $\mathrm{Out}_{3}$. It is visualized in Fig. 2.

Consider a propositional language built from a finite set of propositional atoms, such that all sets of propositions are equivalent to a single formula. A contraction operator for $\mathrm{Out}_{2}$ can be defined as follows.

$R \ominus(a, x)=\operatorname{out}_{2}\left(\left\{(b, y) \in \bar{R} \mid b\right.\right.$ complete, if $b \equiv V(a)$ then $\left.\left.y \in o_{2}(R, V(a))-x\right\}\right)$

where $V(a)$ is a function giving a complete formula implying $a$, and - is an AGM contraction function. 


\begin{tabular}{|c|c|c|c|}
\hline & $\frac{\bar{R}=}{\{(p r, h s e)\}}$ & $\begin{array}{c}\overline{R_{1}}=\bar{R} \ominus(p r \wedge \text { old }, h s e)= \\
\frac{\{(p r \wedge \neg o l d, h s e)}{(p r \wedge \text { old } \wedge y n g, h s e)\}}\end{array}$ & 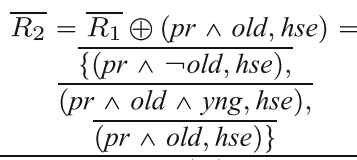 \\
\hline $\mathrm{T}$ & $C n(\top)$ & $C n(\top)$ & $C n(\top)$ \\
\hline$p r$ & $C n(h s e)$ & $C n(\top)$ & $C n(\top)$ \\
\hline$\neg p r$ & $C n(\top)$ & $C n(\top)$ & $C n(\top)$ \\
\hline$p r \vee$ old & $C n(\top)$ & $C n(\top)$ & $C n(\top)$ \\
\hline$p r \wedge$ old & Cn (hse) & $C n(\top)$ & Cn (hse) \\
\hline$p r \wedge \neg$ old & $C n(h s e)$ & Cn (hse) & Cn (hse) \\
\hline$p r \wedge$ old $\wedge y n g$ & $C n(h s e)$ & Cn (hse) & Cn (hse) \\
\hline
\end{tabular}

Fig. 2 Recovery. $(R \ominus(p r \wedge$ old, hse $) \oplus(p r \wedge$ old, hse $)$. We recall the abbreviations used in the figures: low rent ( $h s e$ ), low income agents $(p r)$, free health insurance $(h i)$, elderly agents (old), and young agents (yng)

\subsection{R-6: Extensionality}

K-6: If $\vdash x \leftrightarrow y$ then $K-x=K-y$

(extensionality)

R-6: If $\operatorname{out}(\{(a, x)\})=\operatorname{out}(\{(b, y)\})$ then $\bar{R} \ominus(a, x)=\bar{R} \ominus(b, y)$ (extensionality)

It is the content of $x$ rather than its particular linguistic formulation that determines the contraction of $K$ by $x$. This means that logically equivalent sentences should lead to identical revisions.

The extensionality postulate for rule contraction says that if $\operatorname{out}(\{(a, x)\})=$ $\operatorname{out}(\{(b, y)\})$ then $\bar{R} \ominus(a, x)=\bar{R} \ominus(b, y)$. In other words, if for all $c$, we have $\operatorname{out}(\{(a, x)\}, c)=\operatorname{out}(\{(b, y)\}, c)$, then we have for all $c$ :

$$
\operatorname{out}(\{(a, x)\} \ominus(b, y), c)=\operatorname{out}(\{(b, y)\} \ominus(b, y), c)
$$

\section{Rule Revision}

In this section we discuss rule revision. We start by recalling the AGM postulates for revision, and we then introduce the corresponding postulates for rule revision.

\subsection{Postulates for Rule Revision}

Definition 19 (Alchourrón et al. 1985) Let $K$ be a deductively closed set. An AGM revision operation $*$ satisfies the following postulates.

$\mathrm{K} * 1: K * x$ is a deductively closed set

(closure or type)

$\mathrm{K} * 2: x \in(K * x)$

(success)

$\mathrm{K} * 3: K * x \subseteq K+x$

$\mathrm{K} * 4$ : If $\neg x \notin K$ then $K+x=K * x$

(vacuity)

$\mathrm{K} * 5: K * x=K_{\perp}$ iff $\vdash \neg x$

(triviality)

$\mathrm{K} * 6$ : If $\vdash x \leftrightarrow y$ then $K * x=K * y$

(extensionality) 
$\mathrm{K} * 7: K *(x \wedge y) \subseteq(K * x)+y$

(iterated $K * 3$ )

$\mathrm{K} * 8$ : If $\neg y \notin K * x$ then $(K * x)+y \subseteq K *(x \wedge y)$

(iterated $\mathrm{K} * 4$ )

The same argument as for contraction applies to the last two revision postulates. Hence, we restrict ourselves to the first six postulates. The corresponding postulates for rule revision are the following.

Definition 20 (provisional) Let out be an input/output logic, and $\bar{R}$ a set of rules closed under out. A rule revision operator $\circledast$ satisfies the following postulates.

$\mathrm{R} \circledast 1: \bar{R} \circledast(a, x)$ is closed under out

(closure or type)

$\mathrm{R} \circledast 2:(a, x) \in(\bar{R} \circledast(a, x))$

(success)

$\mathrm{R} \circledast 3: \bar{R} \circledast(a, x) \subseteq \bar{R} \oplus(a, x)$

(inclusion)

$\mathrm{R} \circledast 4:$ If $\neg(a, x) \notin \bar{R}$ then $\bar{R} \oplus(a, x)=\bar{R} \circledast(a, x)$

(vacuity)

$\mathrm{R} \circledast 5: \bar{R} \circledast(a, x)=\bar{R}_{\perp}$ iff $\neg(a, x) \in \operatorname{out}(\emptyset)$

(triviality)

$\mathrm{R} \circledast 6$ : If $\operatorname{out}(\{(a, x)\})=\operatorname{out}(\{(b, y)\})$ then $\bar{R} \circledast(a, x)=\bar{R} \circledast(b, y)$ (extensionality)

Closure, success and extensionality state for revision the homonymous conditions we have seen for theory and rule contraction. $\mathrm{R} \circledast 3$ and $\mathrm{R} \circledast 4$ define the relation between revision and expansion: When $\neg(a, x) \notin \bar{R}$, revision and expansion coincide, i.e. $\bar{R} \circledast(a, x)=\bar{R} \oplus(a, x)$. More generally, the result of revising $\bar{R}$ by $(a, x)$ should contain the new rule together with all those rules that have not been excluded from $\bar{R}$ in order to accommodate $(a, x)$, hence $\bar{R} \circledast(a, x)$ should be a subset of $\bar{R} \oplus(a, x)$, as the inclusion condition states. Finally, $\mathrm{R} \circledast 5$ says that the revision of a normative system should be coherent if the new rule is not contradictory.

However, $\neg(a, x)$ and $\bar{R}_{\perp}$ are not defined in input/output logic thus far. We propose to render postulates $R \circledast 4$ and $R \circledast 5$ as shown below (for completeness we rewrite the complete set of postulates for rule revision):

Definition 21 Let out be an input/output logic, and $\bar{R}$ a set of rules closed under out. A rule revision operator $\circledast$ satisfies the following postulates.

$\mathrm{R} \circledast 1: \bar{R} \circledast(a, x)$ is closed under out

(closure or type)

$\mathrm{R} \circledast 2: \quad(a, x) \in(\bar{R} \circledast(a, x))$

(success)

$\mathrm{R} \circledast 3: \bar{R} \circledast(a, x) \subseteq \bar{R} \oplus(a, x)$

(inclusion)

$\mathrm{R} \circledast 4:$ If $(a, \neg x) \notin \overline{R \cup(a, x)}$ then $\bar{R} \oplus(a, x)=\bar{R} \circledast(a, x)$

(vacuity)

$\mathrm{R} \circledast 5: \quad(a, \neg x) \in \bar{R} \circledast(a, x)$ iff $(a, \neg x) \in \operatorname{out}(\emptyset)$

(triviality)

$\mathrm{R} \circledast 6$ : If $\operatorname{out}(\{(a, x)\})=\operatorname{out}(\{(b, y)\})$ then $\bar{R} \circledast(a, x)=\bar{R} \circledast(b, y)$ (extensionality)

\subsection{From Revision to Contraction (and back)}

Postulates for (belief and rule) revision and (belief and rule) contraction are independent. No reference to contraction is made in the revision postulates, and also the revision operator does not appear in the postulates for contraction. The question of whether belief revision (resp. contraction) could be defined in terms of belief contraction (resp. revision) was addressed in the AGM literature, leading to two positive answers. The Levi identity defines the revision $K * A$ as a sequence of contraction and expansion: 


$$
K * A=(K-\neg A)+A
$$

The correctness of this definition was proven by the theorem below, where $R(-)$ is the revision function obtained from a contraction function with the help of the Levi identity.

Theorem 6 (Gärdenfors and Rott 1995) If a contraction function - satisfies $(K-1)$ to $(K-4)$ and $(K-6)$, then $R(-)$ satisfies $(K * 1)-(K * 6)$.

It is worth mentioning that the controversial recovery postulate $(K-5)$ was not used in the theorem on contraction belief function above, and the fact that it is missing is not due to the problems mentioned earlier for norm contraction. We now show that the same can be done for rule change.

Theorem 7 Given a rule contraction operator, we can define a rule revision operator via the Levi identity, $R(\ominus): \bar{R} \circledast(a, x)=(\bar{R} \ominus(a, \neg x)) \oplus(a, x)$, and when $\ominus$ satisfies $(R-1)$ to $(R-4)$ and $(R-6)$, then $R(\ominus)$ satisfies $\left(R^{*} 1\right)-\left(R^{*} 6\right)$.

We first need to show some lemmas:

Lemma $2 \operatorname{out}(\{(a, x)\})=\operatorname{out}(\{(b, y)\})$ iff out $(\{(a, \neg x)\})=\operatorname{out}(\{(b, \neg y)\})$, when out is out $1-$ out $_{3}^{+}$.

Proof (sketch) In out ${ }_{1}-$ out $_{3}$ it follows from the fact that $\operatorname{out}(\{(a, x)\})=\operatorname{out}(\{(b, y)\})$ iff $C n(a)=C n(b)$ and $C n(x)=C n(y)$. In $o u t_{1}^{+}-o u t_{3}^{+}$(throughput), it follows from the fact that $\operatorname{out}(\{(a, x)\})=\operatorname{out}(\{(b, y)\})$ iff $C n(a)=C n(b)$ and $C n(a \wedge x)=$ $C n(b \wedge y)$.

\section{Lemma 3}

a. If $(a, \neg x) \notin \operatorname{out}(R)$ then $(a, \neg x) \notin \operatorname{out}(R \cup(a, x))$.

b. If $(a, x) \notin \operatorname{out}(R)$ then $(a, x) \notin \operatorname{out}(R \cup(a, \neg x))$.

Proof The two cases are analogous. We only give the first one.

Proof by contradiction. Let us suppose that $(a, \neg x) \in \operatorname{out}(R \cup(a, x))$. Then both the following hold: $(a, \neg x) \in \operatorname{out}(R \cup(a, x))$ and $(a, x) \in \operatorname{out}(R \cup(a, x))$. This would make the set $\operatorname{out}(R, a)$ inconsistent. Unless $x$ is inconsistent, $\neg x$ could not be obtained from $\operatorname{out}(R \cup(a, x), a)$. Thus $(a, \neg x)$ had to be already in out $(R)$ which contradicts the hypothesis.

We can now prove Theorem 7.

Proof $(\mathrm{R} \circledast 1)$ By definition, $\oplus$ leads to the closure under out.

$(\mathrm{R} \circledast 2)$ By definition of $\oplus,(a, x) \in(\bar{R} \circledast(a, x))$.

$(\mathrm{R} \circledast 3)$ By $(R \ominus 2)$ we have that $\bar{R} \ominus(a, x) \subseteq \bar{R}$. We therefore have the following: $\bar{R} \circledast(a, x)=(\bar{R} \ominus(a, \neg x)) \oplus(a, x) \subseteq \bar{R} \oplus(a, x)$.

$(\mathrm{R} \circledast 4)$ Assume that $(a, \neg x) \notin \bar{R} \cup(a, x)$. By $(R \ominus 3)$ we have $\bar{R} \ominus(a, \neg x)=\bar{R}$ and so $\bar{R} \circledast(a, x)=\bar{R} \oplus(a, x)$. 
$(\mathrm{R} \circledast 5)$ Direction $\Leftarrow$. Suppose that $(a, \neg x) \notin$ out $(\emptyset)$. By $(R \ominus 4)$ we have that $(a, \neg x) \notin \bar{R} \ominus(a, \neg x)$, and by Lemma 3.a: $(a, \neg x) \notin(\bar{R} \ominus(a, \neg x) \oplus(a, x))=$ $\bar{R} \circledast(a, x)$. Thus: $(a, \neg x) \notin \bar{R} \circledast(a, x)$.

Direction $\Rightarrow$. out is monotonous in $R,{ }^{2}$ so if $(a, \neg x) \notin(\bar{R} \circledast(a, x))$, then it is also $(a, \neg x) \notin$ out $(\emptyset)$.

$(\mathrm{R} \circledast 6)$ Assume that out $(\{(a, x)\})=$ out $(\{(b, y)\})$. By Lemma 2 , out $(\{(a, x)\})=$ out $(\{(b, y)\})$ iff out $(\{(a, \neg x)\})=$ out $(\{(b, \neg y)\})$, when out is out out $_{1}-$ out $_{4}$ Then by $(R \ominus 6)$ we have that: $\bar{R} \ominus(a, \neg x)=\bar{R} \ominus(b, \neg y)$. So: $(\bar{R} \ominus(a, \neg x)) \oplus(a, x)=$ $(\bar{R} \ominus(b, \neg y)) \oplus(b, y)$, which is: $\bar{R} \circledast(a, x)=\bar{R} \circledast(b, y)$.

Not only belief revision can be defined in terms of belief contraction operators, but also belief contractions can be defined in terms of belief revisions. In the belief change literature, this was expressed by the Harper identity:

$$
K-A=K \cap K * \neg A .
$$

Let $C(*)$ be the contraction function obtained from a revision function $*$ with the help of the Harper identity. As for the Levi identity, that this definition is correct rests upon the following theorem.

Theorem 8 (Gärdenfors and Rott 1995) If a revision function $*$ satisfies $(K * 1)$ to $(K * 6)$, then $C(*)$ satisfies $(K-1)$ to $(K-6)$.

In general a similar relation does not hold for rule change, because we have shown that sometimes (for out 1 and out 3 ) the revision postulates are consistent and the contraction postulates are not. ${ }^{3}$

The natural question now is how these two identities are combined. In the context of AGM theory, Gärdenfors and Rott well explained this point:

The two theorems show that the defined revision and contraction functions have the right properties. But we also want the two definitions to be interchangeable in the sense that if we start with one definition to construct a new contraction (or revision) function and after that use the other definition to obtain a revision (or contraction) function, then we ought to get the original function back. [(Gärdenfors and Rott 1995, p. 57)]

The Levi and the Harper identities have been shown to be interchangeable for AGM theory:

$$
\begin{aligned}
K * A & =(K \cap K * A)+A \\
K-A & =K \cap((K-A)+\neg A) .
\end{aligned}
$$

Hence, transposing the same line of argument for the revision and contraction of rules, and even though there is no theorem corresponding to Theorem 8 in the general

\footnotetext{
2 This follows directly from Proposition 1, see also Makinson and Torre (2000).

3 However, there may be special cases where it holds, for example for out 2 .
} 
case, we can ask whether similar identities hold for rule change. In other words: can we replace $(\bar{R} \ominus(a, \neg x))$ in the Levi identity by $\bar{R} \cap((\bar{R} \circledast(a, x))$ thanks to the Harper identity? And, viceversa, can we substitute $\bar{R} \circledast(a, \neg x))$ in the Harper identity by $(\bar{R} \ominus(a, x)) \oplus(a, \neg x))$ thanks to the Levi identity?

We thus want to check whether $\bar{R} \circledast(a, x)=(\bar{R} \cap \bar{R} \circledast(a, x)) \oplus(a, x)$ is a consequence of the basic postulates for rule revisions, and if $\bar{R} \ominus(a, x)=\bar{R} \cap((\bar{R} \ominus$ $(a, x)) \oplus(a, \neg x))$ can be proved from the basic set of postulates for rule contractions (including the recovery postulate). The answer to the first question is positive:

Proposition $3 \bar{R} \circledast(a, x)=(\bar{R} \cap \bar{R} \circledast(a, x)) \oplus(a, x)$

Proof We show that the following two hold:

- Case 1. $(\bar{R} \circledast(a, x)) \subseteq(\bar{R} \cap(\bar{R} \circledast(a, x)) \oplus(a, x))$. Clearly, $(\bar{R} \cap(\bar{R} \circledast(a, x)) \subseteq$ $(\bar{R} \circledast(a, x))$. When we add $(a, x)$ to the left part, we obtain a set that (like the right part) includes at least $(a, x)$ as out is a consequence operator, as in the right part. The inclusion relation is therefore not reversed. We have that $(\bar{R} \cap(\bar{R} \circledast$ $(a, x)) \oplus(a, x)) \subseteq(\bar{R} \circledast(a, x))$. In the last case we show that $(\bar{R} \cap(\bar{R} \circledast(a, x)) \oplus$ $(a, x)) \nsubseteq(\bar{R} \circledast(a, x))$. So, the two sets must be identical.

- Case 2. $(\bar{R} \cap(\bar{R} \circledast(a, x)) \oplus(a, x)) \subseteq(\bar{R} \circledast(a, x))$. This follows from the inclusion postulate $(R \circledast 3)$, and the fact that out is a consequence operator.

However, $\bar{R} \ominus(a, x)=\bar{R} \cap((\bar{R} \ominus(a, x)) \oplus(a, \neg x))$ does not hold in general, i.e. it cannot hold for out $_{1}$ or out $_{3}$.

\section{Related Work}

Even though Makinson and van der Torre's input/output logic allows to model general input/output processes (as in this paper), so far it has been applied mainly to normative and legal systems. So, the use of input/output logic to AGM theory to capture norm dynamics has been independently pursued by Boella et al. (2009) and Stolpe (2010). Stolpe (2010) analysed derogation and amendment in terms respectively of contractions and revision on a set of norms. When the new norm is incoherent with the existing ones, we have an amendment of the code: in order to coherently add the new regulation, we need to reject those norms that conflict with the new norm. Derogation, on the other hand, is the elimination of a norm together with whatever part of the code that implies that norm. The derogation operation is characterised as an AGM partial meet contraction by defining a selection function for a set of norms in input/output logic. Amendment on the other hand, is defined as a norm revision obtained via the Levi identity. So defined, Stolpe shows that derogation and amendment operators are in one-to-one correspondence with the Harper and Levi identities as inverse bijective maps.

Makinson and van der Torre define their 'operational semantics' of input/output logic (Definition 2 of this paper) out $: \mathcal{P}(L \times L) \times \mathcal{P}(L) \rightarrow \mathcal{P}(L)$, i.e. a context is a (possibly infinite) set of sentences of $L$. In contrast to Makinson and van der Torre and as already discussed in Sect. 2.1, in this paper we are primarily interested in the proof theory of input/output logic, which we use to define what we call here 
rule 'implication.' For this reason we do not consider contexts which may contain infinite sets, and the relation between the semantics and the proof theory is simplified. Likewise, in contrast to Makinson and van der Torre we assume that the language is based on a finite set of propositions, such that the semantics of out $_{2}$ can be based on maxiconsistent formulas rather than maxiconsistent sets.

Governatori and DiGiusto (1999) consider finite base revision (the revision of not deductively closed sets) where facts are distinguished from rules. They study a procedure which defines the revision of a base by a new fact as a change of the rules part of the base: new rules are found in order to accommodate the new information.

Not directly related to norm change is the approach in Corapi et al. (2011), which uses inductive logic programming in order to revise rules and synthesise new ones. The idea is to have a set of use cases defined by the designer's, providing the desired outcome, and thus such defining a semi-automatic process to detect incompleteness and errors. Among the possible extensions mentioned by the authors is also the possibility to adapt such approach to compute revisions of normative frameworks.

In Delgrande et al. 2008; Delgrande 2010 belief revision has been reformulated to capture the change of (non monotonic) logic programs under answer set semantics. A logic program $P$ (i.e. a finite set of rules) is taken to give an agent's epistemic state, whereas the answer sets of $P$ correspond to a belief set in the AGM tradition. Belief revision turns out to be an intuitive approach that can be well suited to capture the revision of logic programs under the answer set semantics, even though the authors do not claim this to be the only possible (or best) approach.

\section{Summary and Outlook}

To apply the AGM theory to rule change, we use input/output logics to define a notion of implication between rules. This notion of implication between rules is based on an 'operational semantics' of a set of rules to derive formulas.

In the tradition of theory change, we have taken contraction to be the more elementary kind of change. For rule base contraction, we show that two existing representation results carry over to rule contraction. However, in this framework, a contraction comes down to a selection of a set of rules, whereas we also like to consider change of the rules. This motivates the study of AGM rule contraction. We also show that for internal and external rule revision we need to adapt Hansson's definitions, as they make use of a disjunction of rules, and negation of rules.

For AGM contraction, we show that the basic postulates can easily carry over to the rule case, but the two additional postulates do not seem to have a straightforward counterpart in rule change. We illustrate that the success postulate may be interpreted as a set of success conditions on outputs. Then we show the surprising result that for two common systems for rules, called simple-minded and reusable output, the postulates do not allow for any rule change operation. On the positive side, we show that rule change operations exist for basic output. We also show that the proof theory of rule change is closely related to the proof theory of permissions from an input/output perspective. 
Finally we consider rule revision, where we only give a partial answer to the question how rule revision methods can be classified. The translation of the AGM postulates is much more difficult than for the contraction case, because we have to define when a set of rules is "consistent", which in the area of normative systems is usually called coherent. We therefore define rule revision in terms of rule contraction using the Levi identity, and we show the the operator thus defined satisfies the AGM rule postulates.

Rules may also be interpreted as orders of ideality, thus introducing preferences over the set of rules. A rule $(a, x)$ would then mean that in context $a, x$ is the most ideal situation. In the literature on belief revision, several proposals for minimal change approaches have been put forward (see, for example, Grove's systems of sphere Grove 1988). We leave the exploration of such connections for future work.

Acknowledgments Thanks to David Makinson and Jörg Hansen for discussions on the issues raised in this paper. We also thank the two anonymous referees for their valuable comments and suggestions that helped us improving the content and readability of the paper. Gabriella Pigozzi benefited from the support of the project AMANDE ANR-13-BS02-0004 of the French National Research Agency (ANR).

\section{References}

Alchourrón, C. E., \& Makinson, D. (1981). Hierarchies of regulations and their logic in Hilpinen, pp. $125-148$.

Alchourrón, C. E., \& Makinson, D. (1982). On the logic of theory change: Contraction functions and their associated revision functions. Theoria, 48, 14-37.

Alchourrón, C. E., Gärdenfors, P., \& Makinson, D. (1985). On the logic of theory change: Partial meet contraction and revision functions. Journal of Symbolic Logic, 50(2), 510-530.

Billington, D., Antoniou, G., Governatori, G., \& Maher, M. (1999). Revising non-monotonic belief sets: The case of defeasible logic. In: KI-99: Advances in artificial intelligence, Berlin: Springer, pp. 101-112.

Boella, G., Pigozzi, G., \& van der Torre, L. (2009). Normative framework for normative system change. In: 8th International joint conference on autonomous agents and multiagent systems (AAMAS 2009), Budapest, Hungary, May 10-15, vol. 1, pp. 169-176.

Corapi, D., De Vos, M., Padget, J., Russo, A., \& Satoh, K. (2011). Norm refinement and design through inductive learning. In M. De Vos, N. Fornara, J. Pitt, \& G. Vouros (Eds.), Coordination, organizations, institutions, and norms in agent systems VI (vol. 6541, pp. 77-94). Lecture Notes in Computer Science Berlin Heidelberg: Springer.

Delgrande, J. (2010). A program-level approach to revising logic programs under the answer set semantics. Theroy and Practice of Logic Programming, 10, 565-580.

Delgrande, J., Schaub, T., Tompits, H., \& Woltran, S. (2008). Belief revision of logic programs under answer set semantics. In G. Brewka, J. Lang (Eds.), (pp. 411-421). KR: AAAI Press.

Gärdenfors, P. (1978). Conditionals and changes of belief. Acta Philosophica Fennica, 30(1), 381-404.

Gärdenfors, P., \& Rott, H. (1995). Belief revision. In: D. M. Gabbay, C. J. Hogger, \& J. Robinson (Eds.) Handbook of logic in artificial intelligence and logic programming. vol. IV: Epistemic and temporal reasoning. Oxford: Oxford University Press, pp. 35-132.

Governatori, G., \& DiGiusto, P. (1999). Modifying is better than deleting: A new approach to base revision. In: E. Lamma, \& P. Mello (Eds.) AI*IA 99, Pitagora, pp. 145-154.

Governatori, G., \& Rotolo, A. (2010). Changing legal systems: Legal abrogations and annulments in defeasible logic. Logic Journal of IGPL 18(1):157-194, http://jigpal.oxfordjournals.org/cgi/content/abstract/ jzp075v1

Governatori, G., Rotolo, A., Olivieri, F., \& Scannapieco, S. (2013). Legal contractions: a logical analysis. In E. Francesconi\& B. Verheij (Eds.) (pp. 63-72). ACM: ICAIL.

Grove, A. (1988). Two modellings for theory change. Journal of Philosophical Logic, 17, 157-170.

Hansson, S. (1993). Reversing the Levi identity. Journal of Philosophical Logic, 22, 637-669.

Makinson, D., \& van der Torre, L. (2000). Input-output logics. Journal of Philosophical Logic, 29, 383-408. 
Makinson, D., \& van der Torre, L. (2001). Constraints for input-output logics. Journal of Philosophical Logic, 30(2), 155-185.

Makinson, D., \& van der Torre, L. (2003). Permissions from an input-output perspective. Journal of Philosophical Logic, 32(4), 391-416.

Nute, D. (1984). Conditional logic. In: Handbook of philosophical logic, synthese library, vol. 165, Berlin: Springer, pp. 387-439.

Parent, X., \& van der Torre, L. (2013). Input/output logics. In D. Gabbay, J. Horty, X. Parent, R. van der Meyden, \& L. van der Torre (Eds.), Handbook of deontic logic and normative systems (pp. 499-544). London: College Publications.

Stolpe, A. (2010). Norm-system revision: Theory and application. Artificial Intelligence and Law, 18, 247-283. 